\title{
Le futur appartient à la jeunesse
}

Aperçus sur l'histoire et l'évolution du mouvement wagnérien des années 1870 à nos jours

\section{Eva Märtson et Philippe Olivier}

\section{OpenEdition}

\section{Journals}

Édition électronique

URL : https://journals.openedition.org/rbnu/2317

DOI : $10.4000 /$ rbnu. 2317

ISSN : 2679-6104

Éditeur

Bibliothèque nationale et universitaire de Strasbourg

\section{Édition imprimée}

Date de publication : 1 mai 2013

Pagination : $30-37$

ISBN : 9782859230432

ISSN : 2109-2761

Référence électronique

Eva Märtson et Philippe Olivier, "Le futur appartient à la jeunesse », La Revue de la BNU [En ligne], 7 | 2013, mis en ligne le 01 mai 2013, consulté le 08 août 2021. URL : http://journals.openedition.org/ rbnu/2317; DOI : https://doi.org/10.4000/rbnu.2317

\section{(c) (1) (-)}

La Revue de la BNU est mise à disposition selon les termes de la Licence Creative Commons Attribution - Pas d'Utilisation Commerciale - Partage dans les Mêmes Conditions 4.0 International. 


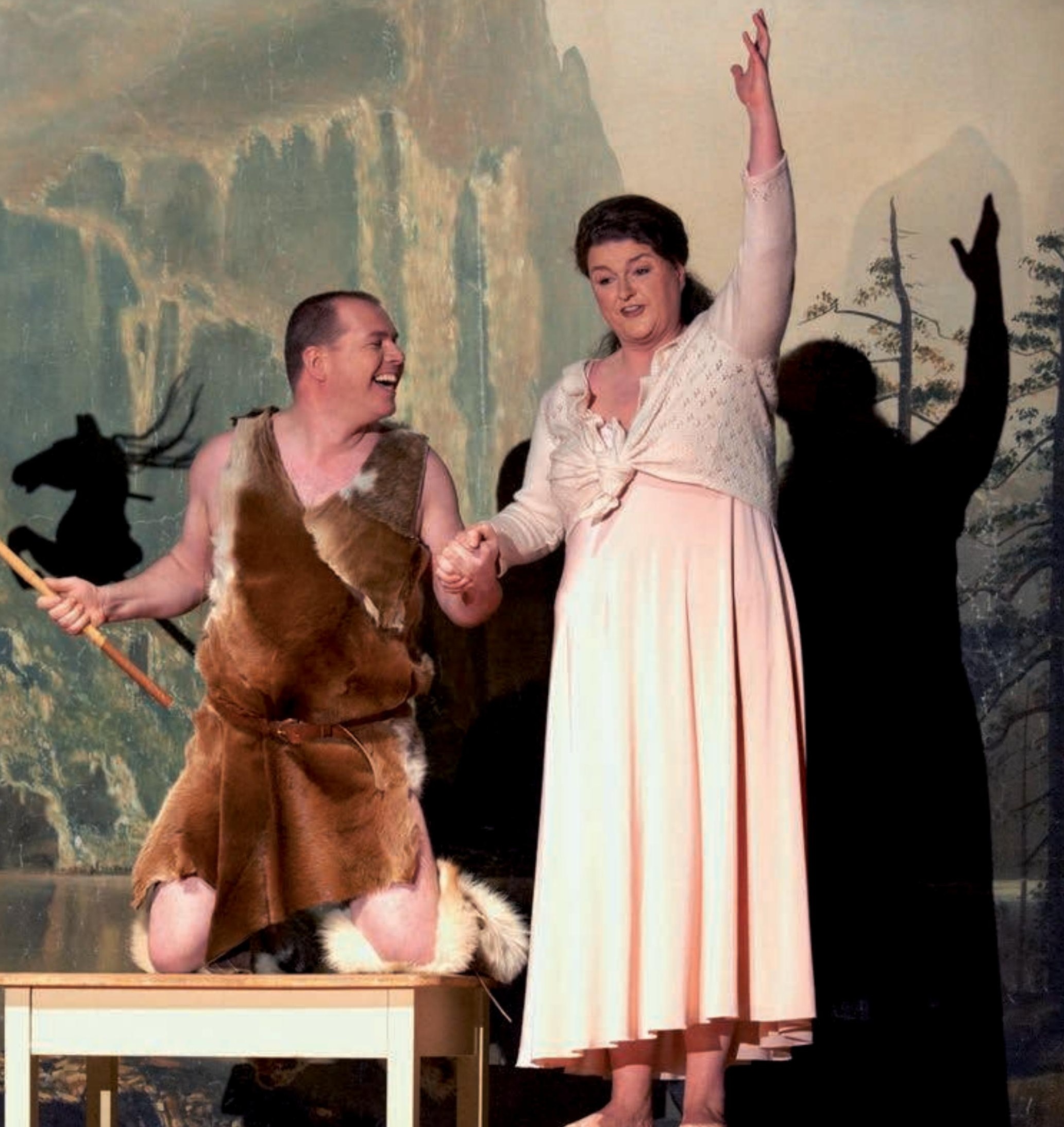




\title{
Le futur appartient à la jeunesse
}

\author{
Aperçus sur l'histoire et l'évolution du mouvement wagnérien \\ des années 1870 à nos jours
}

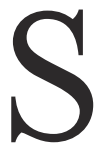

'il existe, de par le monde, des associations internationales vouées à la connaissance, à la défense et à l'illustration de compositeurs comme Wolfgang Amadeus Mozart, Hector Berlioz, Anton Bruckner ou encore Gustav Mahler, ce qu'il est convenu de nommer le " mouvement wagnérien " se distingue par une singularité évidente. Les passionnés du démiurge de Bayreuth se rassemblent, en effet, autour d'une personnalité particulièrement complexe, dont l'influence ne se limite pas à l'art lyrique. Ils ont contribué, à l'origine, au lancement du Festival de Bayreuth. Ils continuent à s'y rendre comme on allait jadis en pèlerinage. Thomas Mann ne déclarat-il pas que "Bayreuth est le Lourdes de l'opéra " ? Ils se regroupent en diverses associations, clubs et cercles.

Ce phénomène n'a rien de nouveau. Le premier des Cercles Richard Wagner est fondé en 1871 à Mannheim, alors que le Festspielhaus n'est encore pas sorti de terre ${ }^{1}$. Il faut, à la fois, apporter des fonds au compositeur pour la construction d'un temple théâtral à sa gloire, autant que soutenir une pensée alors nationaliste, s'appuyant sur une mythologie vigoureuse et la magie de la musique de Wagner. Dès 1874 - deux ans avant la tenue du premier Festival de Bayreuth - vingt-cinq cercles existent déjà en Allemagne et à l'étranger, notamment à Bruxelles, Londres, New York, Prague, Pest ou SaintPétersbourg ${ }^{2}$. Dès les années 1880, un Cercle Richard Wagner se voit créé à Strasbourg. On y rend hommage, en 1884, au compositeur décédé l'année précédente, durant une cérémonie se déroulant au foyer du Stadttheater (Théâtre municipal) de la place Broglie ${ }^{3}$. L'univers, singulier en soi, de ces associations est ici et là animé par des femmes au caractère affirmé. Elles sont souvent de nationalité allemande. Le premier Cercle féminin Richard Wagner voit ainsi le jour à Mayence en 1874 .

Il fera des émules. En 1909, des dames de la bourgeoisie se déplacent à Leipzig afin d'y créer le « RichardWagner-Verband deutscher Frauen " (RWVdF ; en français : Association Richard Wagner des femmes allemandes). Elles tentent de rassembler, avec le soutien de la Princesse Cécile de Prusse ${ }^{4}$, un million de marks or, afin de permettre à des personnes méritantes - au premier chef des jeunes mélomanes de condition sociale modeste - d'assister aux liturgies estivales de Bayreuth sans bourse délier. Le RWVdF sera, courant 1933, contrôlé par le $\mathrm{NSDAP}^{5}$ et conduit à collaborer étroitement avec l'organisation " Kraft durch Freude " ${ }^{6}$. Une telle dissémination sur tout le territoire allemand suscitera, entre autres, la naissance - en 1912 - d'un groupe local du RWVdF à Minden 7 , une petite ville de Westphalie. Faisant aujourd'hui partie de l'Association internationale des Cercles Richard Wagner, le club établi à Minden constitue un véritable cas d'espèce. Il est, depuis un siècle, dirigé par des femmes. Son actuelle présidente produit des représentations wagnériennes au Stadttheater de Minden.

Comme quasiment cent cinquante autres cercles (celui implanté à Strasbourg étant aujourd'hui le plus important de France), celui de Minden appartient à une famille universelle. Elle regroupe vingt-trois mille membres, établis dans trente-sept pays du monde. Presque tous les continents sont rassemblés dans l'Union internationale des Cercles Wagner, ou Richard-Wagner-Verband In- 
ternational e. V. ${ }^{8}$ Cette association de droit allemand, constituée à Bayreuth en 2009, est la structure mère dirigeant une mosaïque de langues, d'usages, d'approches del'œuvre et de la personnalité de Richard Wagner. Quoi de plus varié, en effet, que le Cercle d'Honolulu, celui - récemment fondé - de Pékin ou encore celui d'Abou Dhabi ? En quoi les wagnériens de Buenos Aires se distinguent-ils de plusieurs familles turques éprises de Lohengrin ou de Parsifal depuis qu'Abdülaziz - sultan de la Sublime Porte entre 1861 et 1876 - avait décidé d'apporter un concours financier à Richard Wagner pour permettre la construction du Festspielhaus de Bayreuth ? Pourtant, un dénominateur commun les rassemble : l'intérêt pour le théâtre musical wagnérien et la magie irrésistible du Festival de Bayreuth. Comme l'observe avec justesse Rainer Fineske, le président du Cercle Richard Wagner de Berlin, " tous nos regards sont tournés en permanence vers Bayreuth ». D’autres, un rien irrespectueux, ont surnommé Bayreuth le Vatican del'art lyrique. Tout wagnérien digne de ce nom est allé, va, iraunjouràBayreuth.Orl'accès aux représentations du festival - s'il n'a jamais été facile est devenu un véritable casse-

tête pour tous ses spectateurs, qu'ils soient membres ou non des associations affiliées au RWVI. Pourquoi ?

À l'automne 2011, la Cour fédérale des comptes le Bundesrechnungshof - et la Cour des comptes de l'Etat libre de Bavière ont formulé leurs observations relatives à la démocratisation des modalités d'achat des billets. Eva Wagner-Pasquier et Katharina Wagner ${ }^{9}$, les codirectrices du festival, ont ensuite décidé que les deux représentations habituellement réservées à la DGB, la Confédération allemande des syndicats, seraient supprimées - ceci afin de pouvoir disposer d'environ trois mille places supplémentaires. Cependant, celles-ci n'iront pas aux cercles membres du RWVI. Cette nouvelle donne modifie, dès lors, les relations de celui-ci avec Bayreuth ${ }^{10}$.

Quoi qu'il en soit, 2013 sera - à Bayreuth et ailleurs un millésime exceptionnel. On y fêtera, en effet, le bicentenaire de la naissance à Leipzig de Richard Wagner. Le Congrès international annuel des Cercles Richard Wagner se réunira dans la cité saxonne.
D’innombrables initiatives seront menées à bien dans nombre de pays. Le RWVI soutiendra, ainsi, l'exposition Aus gallischer Sicht, montrant le regard porté par deux wagnériens français - Paul Boulet et Pierre Devraigne sur le Festival de Bayreuth entre la fin des années 1930 et le début des années 1970. Cette exposition, réalisée par le Cercle Richard Wagner de Strasbourg à partir de la collection Boulet-Devraigne ${ }^{11}$ désormais propriété de la Bibliothèque nationale et universitaire ${ }^{12}$ de la même ville, sera successivement présentée à la Médiathèque de Bayreuth, à l'ambassade de France à Berlin et à la Médiathèque André Malraux de Strasbourg. On notera, enfin, que le RWVI pourrait être doté - courant 2013 - du statut d'organisation non gouvernementale auprès du Conseil de l'Europe.

Cette nouveauté, attendue avec impatience, témoigne des changements traversés par notre organisation depuis quelques années. Le monde se transformant aujourd'hui à grande vitesse, le RWVI ne saurait rester à l'écart des métamorphoses apportées par la globalisation, les technologies de l'information, l'évolution des goûts du public ou encore les demandes - légitimes - des nouvelles générations. Sinon, il risque de dépérir rapidement, de devenir un instrument anachronique, et donc de ne plus jouer son rôle d'accompagnateur du mouvement wagnérien, tenant compte des fluctuations inévitables du "Zeitgeist ", de l'esprit du temps. Une telle mission est à la fois exaltante et non dépourvue de risques. En effet, un pareil aggiornamento ne saurait être effectué sans procéder à des ajustements indispensables en ce qui concerne les deux thèmes suivants : l'esthétique des mises en scène wagnériennes et le jeune public.

Il convient d'indiquer ici que, depuis l'ouverture du Festival de Bayreuth pendant l'été 1876, les présentations scéniques des œuvres de Richard Wagner n'ont jamais suscité de consensus - aussi fragile soitil - dans le public. La main de fer de Cosima, la veuve du compositeur, régentant le festival entre 1883 et 1908, transforme celui-ci en un dépôt de reliques. Ainsi, la production de Parsifal demeure inchangée pendant... cinquante et un ans, soit entre 1882 et 1933 ! Quand 


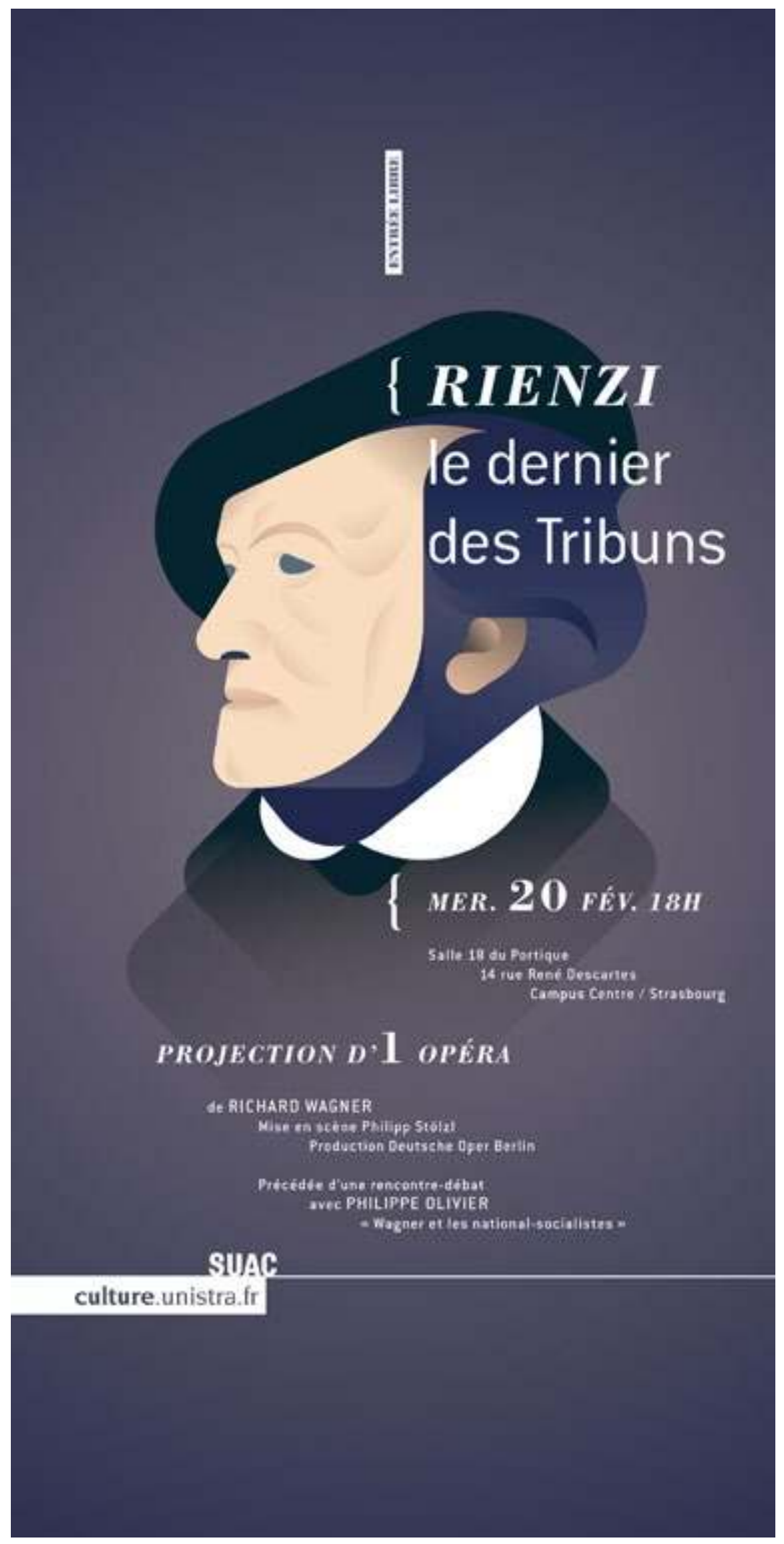

Prospectus réalisé par l'Université de Strasbourg

pour la projection de Rienzi dans ses locaux en février 2013 
Siegfried Wagner, le fils de Richard et de Cosima, ou ses fils Wieland et Wolfgang osent toucher directement ou par metteurs en scène interposés divers éléments symboliques comme la colombe de Parsifal, la ville médiévale des Maîtres chanteurs de Nuremberg ou le jardin nocturne constituant le décor du second acte de Tristan et Isolde, ils sont injuriés, molestés, menacés par le verbe ou la plume, sinon dénoncés à... Adolf Hitler ${ }^{13}$ !

La révolution esthétique opérée par Wieland exaspère aussi une partie des fidèles de Bayreuth, dont Paul Boulet... tout en suscitant l'appui inattendu d'Albert Schweitzer ${ }^{14}$ ! Une fois Wieland décédé, son frère Wolfgang ouvre le Festspielhaus à des metteurs en scène dits " aventureux ». Ils se nomment Patrice Chéreau, Harry Kupfer, Werner Herzog, Heiner Müller ou Christoph Schlingensief. Des négociations avec Luchino Visconti, Peter Stein et Lars von Trier finissent par s'ensabler. Depuis l'arrivée d'Eva Wagner-Pasquier et de Katharina Wagner aux affaires, une modernité impérieuse habite la scène bayreuthienne, avec ses réussites, ses échecs, ses zones d'ombre. Une pareille évolution divise tous les publics, qu'ils ne jurent que par Wagner ou que la palette de leurs goûts couvre d'autres compositeurs. Les membres des cercles Richard Wagner ne sont pas dispensés de prendre part à cette querelle des anciens et des modernes. Ils s'y impliquent, en fonction de leurs goûts personnels. Dans un tel contexte, la mission du RWVI consiste à tenter de maintenir - plus que jamais les plateaux de la balance en équilibre. L'exercice n'est pas aisé. En effet, les signes de l'expression théâtrale évoluent en permanence. Ne faudrait-il pas, à cet égard, confier aux cercles Richard Wagner le soin d'organiser des conférences ou des débats de sensibilisation à ces changements chroniques?

Le désir originel de Richard Wagner, en créant Bayreuth, était d'ouvrir un atelier, une " Werkstätte ", dominée par une volonté d'innovation et de recherche permanentes. Pas un sanctuaire. Dès lors, la Tétralogie devant être présentée sur la " verte colline " - en juillet et en août 2013 - à l'occasion du bicentenaire de sa naissance sera placée sous le signe de la continuité. Son maître d'œuvre sera le metteur en scène Frank Castorf, par ailleurs directeur de la Volksbühne de Berlin depuis 1992. L'esthétique de Castorf est représentative de notre époque ; elle correspond aux attentes d'un nouveau public. Sa récente production de Die Wirtin ${ }^{15}$ présente le désarroi de la jeunesse pauvre d'aujourd'hui, sa liberté de mœurs et la négation permanente des valeurs traditionnelles. À cet égard, rien n'a changé dans le monde depuis Eschyle et l'un de ses continuateurs nommé Richard Wagner. Le Ring montre la trahison et le parjure, l'escroquerie et le mensonge permanents, la violence faite aux femmes, l'inceste ou encore la polygamie de Wotan.

Dès lors se pose une question fondamentale : a-t-on le droit de confisquer la représentation de ces faits et gestes au profit d'une seule partie du public et de l'adapter à sa seule vision de l'univers ? Il semble que, pour certains, le théâtre musical demeure la perpétuation des théâtres de cour de l'Ancien Régime. Ce qui est montré sur scène, des décors et des costumes au moindre accessoire, doit correspondre à leur conception de l'univers. Les artistes sont - avec un pareil état d'esprit - à leur service. On retrouve, en cela, les privilèges d'antan, décrits dès 1876 - l'année de l'ouverture du Festival de Bayreuth ! - par Hippolyte Taine au long des Origines de la France contemporaine. Traitant du contexte général à la veille des événements de 1789 , le penseur originaire des Ardennes écrit : " Après huit cents ans, malgré [...] l'immense changement de la culture sociale, la vieille racine féodale dure et végète toujours ${ }^{16}$.

La démocratie aidant et la jeunesse étant l'avenir du monde, il y a lieu de s'interroger sur le rôle présent et - surtout - futur du RWVI. Doit-il se consacrer à ses seuls adhérents ou intéresser avec détermination les générations nouvelles à l'œuvre et à la figure de Richard Wagner ? Divers éléments de réponse peuvent être ici apportés. Ils sont très contrastés. Ici, certain cercle est manifestement plus intéressé à l'organisation de repas gastronomiques qu'à un travail approfondi sur l'auteur de Parsifal. Là, un autre cercle refuse d'amener ses adhérents voir des spectacles réalisés selon des principes d'avant-garde. Ailleurs, des séminaires d'étude sont organisés régulièrement. Dans une autre ville, on consacre un samedi ou un dimanche entiers à l'analyse du second acte de Tristan et Isolde. Le Cercle Richard Wagner de Munich aide à la création d'un nouveau cercle à La Havane et lui envoie des partitions. Celui de Berlin se déplace à Buenos Aires afin d'assister à la représentation - au Teatro Colón - d'une version condensée du Ring. Celui de Genève prépare, pour le début novembre 2013, un Wagner Festival ${ }^{17}$ qui sera réalisé avec le Grand Théâtre de cette ville. À Graz, le Wagner-Forum organise - entre autres - un concours international de mise en scène d'opéra. Sa prochaine session aura lieu en 2014. Elle permettra, une fois encore, de déceler des talents au style très actuel ${ }^{18}$, d'autant que l'un de ses partisans les plus déterminés est le metteur en scène Peter Kon- 

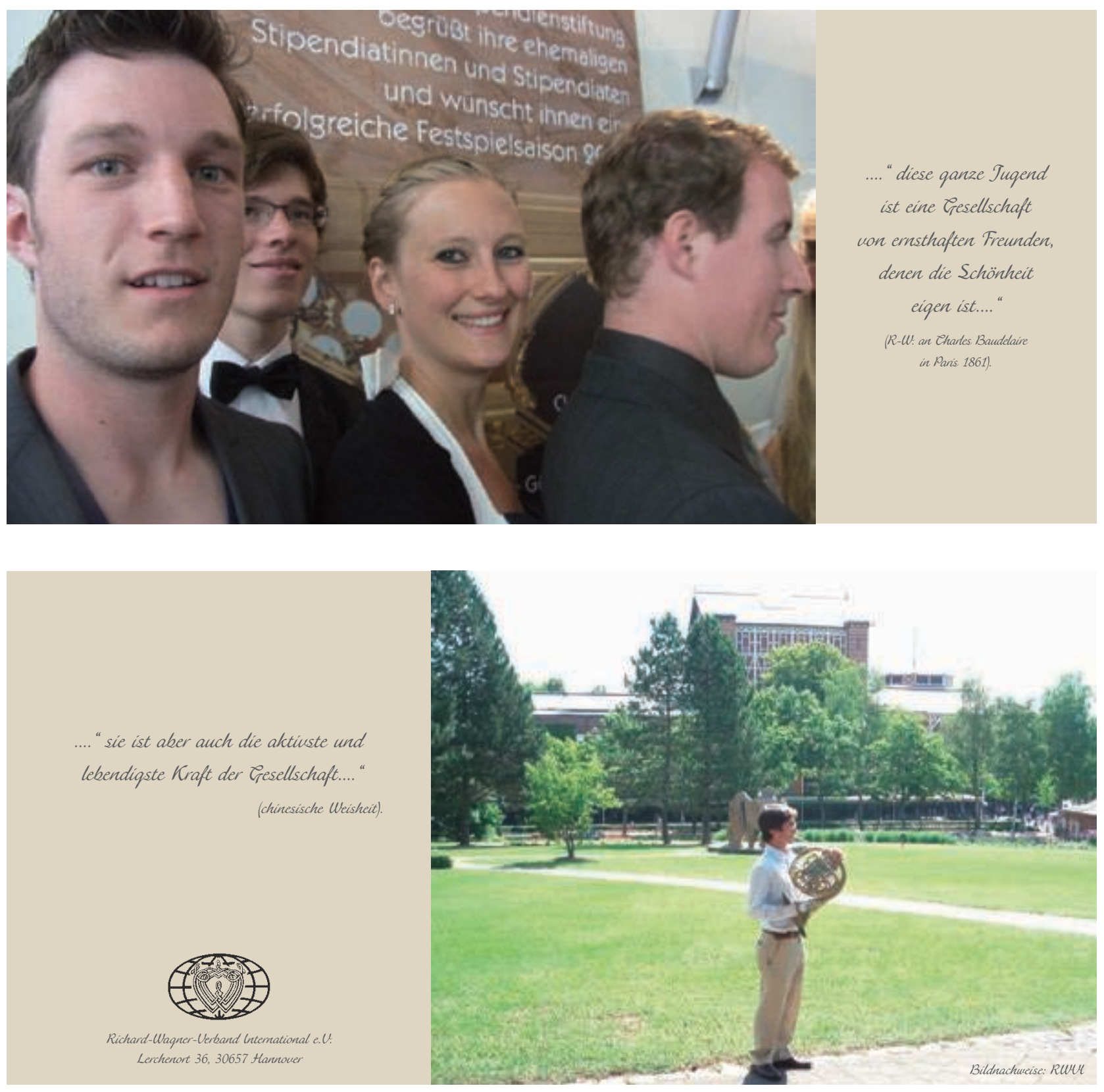

Carte de vœux pour 2013 réalisée par l'Union mondiale des cercles Wagner 


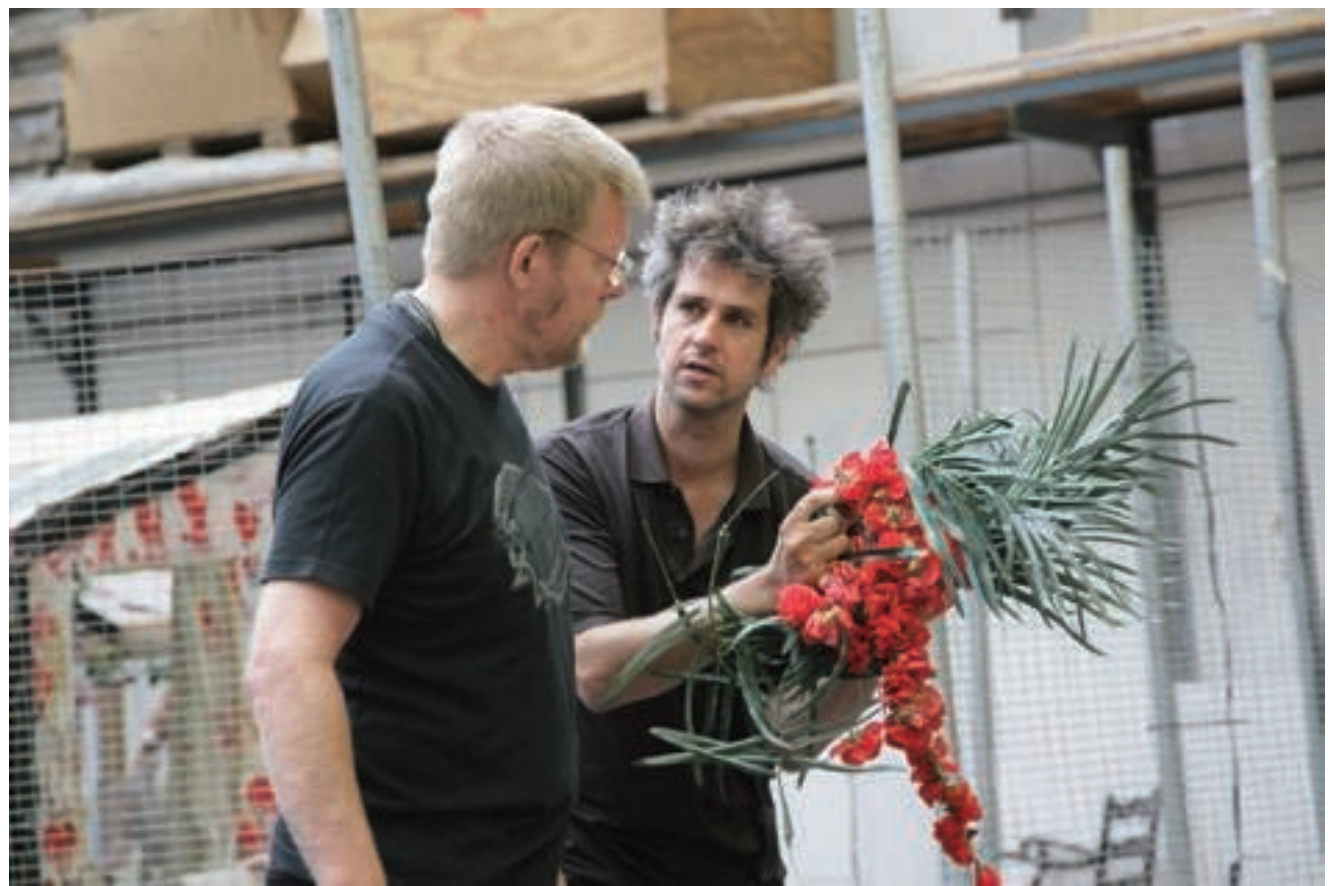

Christoph Schlingensief (à droite) lors d'une répétition (coll. Bayreuther Festspiele)

witschny, qui a réalisé un Crépuscule des dieux devenu légendaire à l'Opéra d'État de Stuttgart en 2000 (déclaré alors meilleur spectacle de l'année en Allemagne par la sévère revue Opernwelt) ${ }^{19}$. À 68 ans, Peter Konwitschny n'a manifestement pas l'âge de ses artères. Son rythme de production est des plus impressionnants. N'a-t-il pas, en décembre 2012, présenté un Faust de Goethe décapant à Graz ?

Peter Konwitschny fait partie des hommes de théâtre qui fascinent les jeunes générations, au même titre que le regretté Christoph Schlingensief ${ }^{20}$ qui a, lui, abondamment utilisé la vidéo dans son Parsifal de Bayreuth présenté entre 2004 et 2006 - où la lutte d'actualité entre les sources chrétiennes occidentales et l'islam soutenait le propos dramaturgique. Il traitait en même temps du vaudou et de son culte du sang, lui-même omniprésent au long du «Bühnenweihfestspiel » de Richard Wagner, en raison de la plaie ayant distingué le flanc de Jésus au Golgotha et de la maladie affectant Amfortas, le roi du Graal. Le même Schlingensief était, au demeurant, fidèle à la problématique fondamentale du " Gesamtkunstwerk » (œuvre d'art totale) wagnérien : " une explication intensive avec les pôles extrêmes de sa dimension utopique [...] : d'une part l'opéra en tant que forme encore et toujours élitaire et, d'autre part, la société " $^{21}$, celle d'autrefois comme celle d'aujourd'hui.

La vidéo et le temps présent : ces thèmes sont évidents pour le jeune public. Ils appartiennent à son rapport au monde, rapport d'aujourd'hui et de demain. La transmission des trésors culturels de notre civilisation - ceux notamment laissés par l'enchanteur de Bayreuth - étant inscrite dans l'objet social même du RWVI, celui-ci a donc le devoir - de plus en plus urgent de former les nouvelles générations. Un tel objectif ne saurait être accompli en ayant recours à des formules éculées ou appartenant au passé. Il convient de trouver les juniors potentiellement intéressés là même où ils se trouvent - à savoir dans le monde de l'enseignement supérieur.

Une nouvelle activité du RWVI, à laquelle nul n'aurait pu songer voici trois ou quatre décennies, est représentée par la création de cercles universitaires. Tel est le cas, par exemple, à l'Université FriedrichAlexander d'Erlangen-Nuremberg, ou encore à Trèves, Sarrebruck, Oxford, Cambridge ou Singapour. Ayant été fondés, pour les premiers d'entre eux, en 2009, ces cercles continueront à se développer en 2013. Les universités de Bonn, de Karlsruhe et de Strasbourg intégreront ainsi un tel dispositif. Des groupes de travail interdisciplinaires y fonctionneront. Les étudiants en chimie pourront - par exemple - s'interroger sur les philtres et autres boissons dans les œuvres de Richard Wagner. Quant aux futurs médecins, ils disposeront de la faculté d'aborder les maladies ayant éprouvé le compositeur. Une telle activité a, au demeurant, été précédée depuis 2009 de séminaires intensifs organisés avec la Fondation Konrad Adenauer. Ils se sont notamment déroulés à Paris ou à Zürich. 
Les doctorants travaillant à des thèses relatives à l'œuvre de Richard Wagner peuvent aussi bénéficier désormais d'une bourse décernée par les instances dirigeantes du RWVI. Tel est le cas de cinq d'entre eux, à savoir quatre Allemands et un étudiant inscrit à l'Université d'Oxford. Il s'agit d'Antonia Goldhammer, de Martin Schneider, d'Eugen Wenzel, de Simone Brandes - qui a soutenu une thèse établissant les relations entre Salvador Dali et Richard Wagner, tout comme d'Henry James Hope. Sans jamais oublier que « les faits ne sont pas l'Histoire " 22 , ces jeunes chercheurs enrichissent l'analyse éternellement en cours de la figure et de l'œuvre de Richard Wagner. Ils méritent, d'ores et déjà, la reconnaissance de leurs aînés : le futur appartient à la jeunesse. Le Présidium du RWVI les encourage de manière concrète, car la musique et la culture unissent les générations.

\section{Eva Märtson et Philippe Olivier}

\section{Notes}

1- Il sera inauguré pendant l'été 1876 , à l'occasion de la création mondiale intégrale de la Tétralogie.

2 - Les armoiries de ces villes orneront le salon se trouvant au rez-de-chaussée de la villa Wahnfried, la résidence de Richard Wagner à Bayreuth.

3- La manifestation se déroula le 27 février 1884. Organisée par la section strasbourgeoise de l'Allgemeiner Richard-Wagner-Verein, elle comportait une allocution et un fragment de Tristan et Isolde, ainsi que le Liebesmahl der Gralsritter de Parsifal chanté par les choristes masculins du Stadttheater. Deux pianos et un harmonium les accompagnaient en lieu et place d'un orchestre.

4- Née en 1886 et disparue en 1954, la Kronprinzessin était une mélomane passionnée. Elle s'engagea aussi nettement en faveur de l'éducation des femmes. Nombre d'écoles et de rues portent encore son nom aujourd'hui en Allemagne.

5- Le Parti national-socialiste allemand des travailleurs (Nationalsozialistische Deutsche Arbeiterpartei)

6- « La force par la joie », en abrégé KdF, omniprésente et omnipuissante organisation pourvoyeuse de loisirs pour les Allemands hitlériens. On constate, en l'espèce, la relation particulière établie entre le Festival de Bayreuth et la politique allemande - tant intérieure qu'étrangère entre 1933 et 1945.

7- Un premier Cercle Richard Wagner y avait été fondé en 1878.

8- En abrégé : RWVI. Cette abréviation sera utilisée dans les pages suivantes. Adresse internet : www.richard-wagner-verband.de

9- Il s'agit des filles de Wolfgang Wagner, lui-même petit-fils du compositeur et décédé en 2010. Ce dernier avait renoncé - en raison de son âge - à diriger le festival en septembre 2008. Ses deux filles lui ont succédé au terme d'une procédure de recrutement ayant particulièrement intéressé les grands médias de nombreux pays. Leur concurrente était leur cousine Nike Wagner - la fille de Wieland Wagner - qui s'était présentée conjointement avec Gérard Mortier, l'ancien directeur du Festival de Salzbourg et de l'Opéra national de Paris.
10 - L'année 2012 aura, à cet égard, été marquée par d'innombrables entremises et correspondances. Les cercles affiliés au RWVI auront, entre autres, adressé une pétition aux sœurs Wagner. Des lettres auront été adressées aux plus hautes autorités de la République fédérale d'Allemagne.

11 - L’arrivée de la collection Boulet-Devraigne dans la métropole alsacienne n’a pas été du goût de certains mélomanes à la fois wagnériens et parisiens. Ils se sont étonnés que cet ensemble de documents rares ne reste pas dans la capitale française. Voir aussi la rubrique « Nouvelles acquisitions patrimoniales ".

12 - Les autres partenaires de l'exposition sont la Ville de Strasbourg, le Conseil régional d'Alsace, le Conseil général du Bas-Rhin, la Fondation Entente franco-allemande, la Banque populaire d'Alsace et Suez environnement, tout comme l'ambassade de France en Allemagne.

13 - Tel fut le sort réservé en 1934 au metteur en scène Heinz Tietjen, comme aux décorateurs Alfred Roller et Emil Preetorius. Ils furent attaqués dans une pétition rédigée par le Suisse Adolf Zinstag et adressée au Chancelier du Reich. Une copie de ce libelle fut transmise à Winifred Wagner, devenue la maitresse du festival depuis la disparition de son mari Siegfried, décédé pendant le festival de 1930.

14 - Philippe Olivier, Der Ring des Nibelungen in Bayreuth von den Anfängen bis heute (préface de Wolfgang Wagner), Schott, Mayence, 2007, p 120. Voir aussi, à propos de Boulet, la rubrique " L'inédit ".

15 - Tiré d'un récit de Dostoïevski, ce spectacle a été présenté pour la première fois le 2 novembre 2012 à la même Volksbühne.

16 - Hippolyte Taine, Origines de la France contemporaine, Robert Laffont, Paris, 2011, p. 17

17- www.wagner-geneva-festival.ch

18 - www.wfg.at

19 - Cette production a été reprise à Stuttgart, sous la direction musicale de Marc Soustrot, en janvier et février 2013. Elle a remporté de nouveau un accueil triomphal. Néanmoins, sa représentation du 3 février aura donné l'occasion de constater - une fois encore - l'opposition opiniâtre de certains spectateurs à la modernité. L'un d'eux, installé au troisième rang des fauteuils d'orchestre, s'est levé pour conspuer le metteur en scène juste avant le début du spectacle. Un autre a quitté bruyamment la salle en proférant des insultes grossières contre Peter Konwitschny. Une troisième personne a eu un malaise au commencement du troisième acte et - une fois rétablie - s'est écriée que le metteur en scène était responsable d'un tel désagrément " tant ses extravagances sont mauvaises pour la santé » ( ?!). L'Opéra d'État de Stuttgart étant au nombre des théâtres lyriques européens les plus avancés en matière de travail théâtral, on imagine les réactions suscitées par une telle production du Crépuscule des dieux dans une institution moins radicale.

20 - Disparu à près de cinquante ans en 2010, Schlingensief a suscité l'éloge funèbre suivant d'Elfriede Jelinek, prix Nobel de littérature : « Il était un artiste par nature ». De surcroît, les provocations auxquelles il s'était livré durant sa carrière ne furent pas sans rappeler celles que les contemporains de Richard Wagner reprochèrent à ce dernier.

21- Anno Mungen in Das Wagner Lexikon (direction : Daniel Brandenburg, Rainer Franke et Anno Mungen), Laaber-Verlag, 2012, p. 263

22 - Yosef Hayim Yerushalmi, Transmettre l'histoire juive (Entretiens avec Sylvie Anne Goldberg), Albin Michel, 2012, p. 185 